\title{
Collectivism, Effects On Relationships
}

Collectivism is a cultural syndrome, a network of norms, values, and ways of engaging the world embedded in practices, artifacts, institutions, language use and structure, and ways of making sense of history. A central theme of collectivism as a cultural syndrome is that relationships with relevant others and group memberships constitute the primary unit of society, the foundation of self-concept, and the key values that should govern one's life. Social units that share a common fate are centrally important from a collectivistic world-view, which highlights interdependence among ingroup members and relevant values, such as loyalty and perseverance. These social units can be broadly defined as groups with which a real or symbolic blood tie exists (families, tribes, races/ ethnicities, religions, nations, peoples) as well as, in some situations, civic (neigh borhood or community) or other working groups.

Within a collectivistic perspective, each social unit feels tangible and real, and group members are obligated to and interdependent with each other. In this way, group memberships and the relationships they entail are permanent, fixed facts of life that are indefinitely obligating. Within a collectivistic perspective, individuals can only be understood within the context of the groups they belong to and in terms of their connections with particular others. Group memberships are assumed to be stable, impermeable, and central to self-concept. Fulfilling one's obligations to group members and collective welfare are therefore central to well-being, while communicating and pursuing one's personal goals and desires is assumed to be at best a secondary issue. The alternative to a collective perspective is an individualistic perspective in which the individual is the most basic unit of society. Individuals are defined by their own attainments and relationships, and group memberships have meaning only insofar as they facilitate the attainment of personal goals. This entry describes theory and research about collectivism and its effects on relationships.

\section{Theoretical Implications of Collectivism: Impact on Relationship, Values, Self- Concept, and Cognition}

A collectivistic worldview should have important consequences for how relationships are conceptualized, what is perceived to be of value, and how the self is conceptualized. When perceived through a collectivistic view, a primary human goal is to maintain important group memberships and the necessary interpersonal relationships that group memberships create. This requires focusing on fitting in and appropriately engaging in social contexts. Figuring out what is appropriate requires skill in indirect communication (reading between the lines of communication and making inferences given context, tone of voice, and other cues) and the ability to restrain oneself, not stick out or offend ingroup others.

To maintain harmony in relationships with ingroup others requires a heightened sensitivity to interpersonal contextual cues including concerns for belongingness, dependency, empathy, norms for reciprocity, and occupying an appropriate place within the relationship hierarchy. These relationships require detailed interpersonal knowledge and constant awareness of others, their needs, desires, and goals. Thus, satisfying one's own need to connect with others may best be met by satisfying others' needs. One perspective on how to fit in and anticipate the needs of ingroup others comes from an EastAsian Confucian-based perspective. From this perspective, individuals are socialized to effectively regulate and control their own emotional displays, smoothing out both displays of negative and positive emotions in settings where either may offend others. Another perspective comes from African, Mediterranean, Middle-Eastern, and Latin perspectives that emphasize ingroup others as important sources of honor. Honor-based collectivism also emphasizes that the self is defined by ingroup others but does not necessarily focus attention on regulation of emotional expression. Rather, in these contexts, displays of anger, hostility, and other strong emotions can be appropriate ways to mend and restore honor. A drawback of research to date on collective world-view and relationships is that it mostly contrasts Eastern (Asian) and Western (especially North American) societies, suggesting that results so far may be limited to a certain kind of collectivism.

Engaging in collectivism-appropriate relationships requires that one's values, goals, and thoughts about the self make sense within these relationships. From a collectivistic perspective, relationships with others are not merely connected to self-concept; they are the central, defining feature of self-concept. Without one's relationships, one would not be the same person. In this way, collectivism is centrally defined by a connection between (rather than a separation from) the self and relevant others.

Indeed, to make sense of gender differences and cross-cultural differences between Eastern (e.g., Japan) versus Western (e.g., United States) societies, researchers have contrasted interdependent and independent ways of defining the self and linked these differences in self-concept to differences in 
cognitive processes. This research finds consistent cross-national differences in relational styles that parallel differences in perception and cognition, how stimuli are perceived, the sense made of information and conclusions drawn from information as well as what feels convincing and worthy of attention.

\section{Assessing Collectivism and Its Effects}

An important early operationalization of collectivism was provided by Geert Hofstede in his 1980 book, Culture's Consequences. He conceptualized a single scale with low individualism (high collectivism) anchoring one end and high individualism (low collectivism) anchoring the other. Hofstede identified this factor by factor-analyzing work-related value questions across 65 nations. In doing so, he organized the myriad possible ways in which countries could differ into a small number of specific factors (with individualism being the first and most widely studied focus of attention). Averaged responses were used to create country scores, which he demonstrated were correlated with nation-level variables such as population size and gross national product.

Although Hofstede did not argue that his findings should be assumed to be fixed in time, since 1980 , others have applied his results in making sense of their own cross-national comparisons, inferring that observed differences in samples drawn from two different nations are due to differences in individualism or collectivism. Indeed, a large number of country-country comparisons find results compatible with the notion that nations differ in collectivism and in individualism. However, there are three main criticisms of this approach and the studies that use it. First, these studies do not lay out the process connecting country-level differences in individualism or collectivism and individual differences in dependent variables of interest such as relational style. Second, they typically demonstrate that differences exist between countries but do not directly link these differences to individualism and collectivism. Third, by inferring that difference is due to individualism and collectivism, these studies cannot separately assess impact of individualism and collectivism. To address these shortcomings, some studies include a direct assessment of individualism and collectivism (study participants are asked to describe their values, attitudes, and norms). This allows for direct assessment of the link between individualism or collectivism and outcomes of interest such as relationship style.

This approach also can be criticized because collectivism is operationalized as a set of responses to value, attitude, and norm questions. A separate literature has shown that self-reports are highly influenced by the context in which they are asked; the answers one gives depend on what seems relevant in the moment and what is relevant can be due to contextual cues one does not even consciously notice. For example, when asked to describe oneself, people are more likely to use personality traits when the study comes from an institute of personality research than from an "institute of social research," even though they are not aware of making this adjustment. In spite of this limitation, research connecting self-reported individualism and collectivism with relationship style has yielded important and theoretically meaningful results, consistent with cross-national comparisons. To examine the overall picture of this research, a quantitative synthesis (meta-analysis) across studies was needed to look for patterns that cannot be seen in a single study. Daphna Oyserman, Heather Coon, and Markus Kemmelmeier performed such a synthesis and suggest three important conclusions. Both individualism and collectivism can be assessed. Countries differ systematically on these measures. Finally, individualism and collectivism scores are not related. That is, high collectivism is not necessarily the same as low individualism.

Why might this be? One possibility is that rather than socialize only for individualism or only for collectivism, societies may actually socialize for both but differ in the situations in which one or the other framework is relevant. Saying that a society is high in collectivism would then simply be a shorthand way of saying that people living in that society usually make sense of themselves, others, and the world within a collectivistic framework but could use an individualistic framework if it seemed relevant in a particular context. A clear example involves engagement with outgroup others. Collectivism does not imply that one engages in a connected and related way with all others, just with ingroup others. When the ingroup is not relevant, one is free to compete with others, express oneself, and follow one's desires.

\section{Empirical Evidence for the Relational Effects of Collectivism (and Individualism)}

In their meta-analytic review, Daphna Oyserman and colleagues identified 71 studies examining assumed or assessed effects of individualism and collectivism on relationships of various kinds. Assumed effect studies used cross-national comparisons rather than directly assessing individualism or collectivism, assuming for example, that if Americans differ from Chinese on a variable interest, the pattern of results can be explained by (unmeasured) differences in individualism or collectivism.

Close relationships were studied in 17 studies, which asked, for example, whether individualistic perspective was associated with romantic love, family obligation, and other close relationships. Effects for romantic love were inconclusive. Similarly, felt closeness and obligation to nuclear family are not a simple function of collectivism. Effects are complicated by the fact that felt obligation can also be perceived as part of a chosen relationship, and this seems to be how Americans tend to view their 
family relationships. Generally, research did not support the notion that Americans have more conflicted family and intimate relations than others but did support the idea that individualism promotes ease of interacting with strangers and more direct communication styles, while collectivism promotes ingroup preference in relationships and different forms of face-saving. Effects were substantial, often moderate to large in size, though highly variable by study and outcome. Study results suggest more freedom to choose interaction partners and lower relationship commitment and engagement with a larger group of less close others when individualism is high, but higher conformity and more time spent with ingroups rather than outgroups when collectivism is high. For example, although interacting with strangers is always more difficult than interacting with ingroup members, Japanese and Koreans report a larger difference than Americans in how they act with ingroup members versus strangers. Americans interact with more people, more frequently, whether individually or in groups, than do Hong Kong-Chinese students.

Another 38 studies examined interactions within and across groups, showing for example that a preference for forming relationships with ingroup others over outgroup others is associated with collectivism. A final 16 studies examined workplace relationships such as supervisor plans to motivate employees. These studies found that American supervisors were more likely to prefer direct communication, individual-level rewards, and tit for tat responses (e.g., immediately reward good behavior and punish bad behavior) rather than respond more indirectly or provide longer-term, grouplevel rewards and responses. Here too the average effects of individualism and collectivism on relationships and relationship styles were substantial.

Of course, the question remains whether these cross-national results are actually due to individualism, collectivism, or perhaps something else. Daphna Oyserman and Wing Sing (Spike) Lee conducted a second meta-analytic review to address this question and found 13 studies examining relevant outcomes (relationship style, felt support, closeness, cooperation, and social obligation). These studies leverage prior research, demonstrating that self-reports and behavior are highly influenced by contextual cues, to prime (or to make momentarily salient) relevant active ingredients of either individualism or collectivism. For example, participants were asked to read a paragraph about a trip to the city that either included the pronouns I, me, and myself (individualism prime) or the pronouns we, us, and ourselves (collectivism prime). Researchers found that on average, the answers participants gave to the relationship questions depended on whether they had just circled singular or plural pronouns. Collectivism-primed participants felt more obligated to help, made more prosocial choices, and were more in favor of affirmative action, suggesting that when made salient in the moment, a collective worldview facilitates greater social obligation and cooperation with others.

Nicholas Sorensen and Daphna Oyserman

\section{Further Readings}

Hofstede, G. (1980). Culture's consequences. Beverly Hills, CA: Sage.

Markus, H. R. and Kitayama, S. Culture and the self: Implications for cognition, emotion, and motivation. Psychological Review vol. 98 (1991). pp. 224-253.

Oyserman, D. , Coon, H. M. , and Kemmelmeier, M. Rethinking individualism and collectivism: Evaluation of theoretical assumptions and meta-analyses. Psychological Bulletin vol. 128 (2002). pp. 3-72.

Oyserman, D. and Lee, S. S. W. Does culture influence how we think: Effects of priming individualism and collectivism. Psychological Bulletin vol. 134 (2008). pp. 311-342.

\section{Entry Citation:}

Sorensen, Nicholas, and Daphna Oyserman. "Collectivism, Effects On Relationships." Encyclopedia of Human Relationships. 2009. SAGE Publications. 2 May. 2011. <http://www.sageereference.com.proxy.lib.umich.edu/humanrelationships/Article_n80.html>.

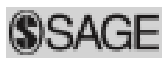

(C) SAGE Publications, Inc. 